\title{
A SPATIO-TEMPORAL WEB APPLICATION FOR THE UNDERSTANDING OF THE FORMATION OF THE PARISIAN METROPOLIS
}

\author{
E. Blettery ${ }^{1,2}$, P. Lecat $^{1}$, A. Devaux ${ }^{2}$, V. Gouet-Brunet ${ }^{2}$, F. Saly-Giocanti ${ }^{1}$, M. Brédif ${ }^{2}$, L. Delavoipière ${ }^{3}$, S. Conord ${ }^{3}$, F. $_{\text {Moret }}{ }^{1}$ \\ ${ }^{1}$ ACP, Univ Gustave Eiffel, 77454 Marne-la-Vallée, France \\ ${ }^{2}$ LASTIG, Univ Gustave Eiffel, ENSG, IGN, 94160 Saint-Mande, France \\ ${ }^{3}$ Univ Paris Nanterre, Mosaïques group, UMR LAVUE (CNRS, 7218), Nanterre, France
}

KEY WORDS: Computer graphics, HCI, Photogrammetry, Aerial imagery, Digital humanities, Urban history, Population census

\begin{abstract}
:
This article presents a spatio-temporal web application dedicated to the co-exploitation of heterogeneous data spatialized in a common 3D environment, providing several paradigms for supporting their co-visualization and interactions within the 3D environment and across time. The relevance of this tool is demonstrated here with two use cases involving historians and sociologists with the common objective of better understanding the formation of the Parisian metropolis. The study focuses on the evolution of the city of Nanterre (Paris area), which underwent many changes in the 1950s, and in particular on shantytown areas. Through census as statistical data and aerial imagery as visual data, a group of historians and sociologists experimented the relevance of the joint exploitation of those heterogeneous data within the proposed spatio-temporal web application.
\end{abstract}

\section{INTRODUCTION}

In recent years, the massive deployment of digitisation technologies and the increasing availability of digital data describing the past have made of those "big data of the past" a major challenge for research in information science and digital humanities. They represent an enormous potential for knowledge and understanding of our social, cultural and geographical heritage and its evolution over time. These data may be very heterogeneous, e.g. statistical data, ancient texts, iconography, 3D objects, etc. They are usually distributed in silos in diverse GLAM (Galleries, Libraries, Archives, Museums) institutions and poorly interconnected. Their richness, facing their variety, complexity and lack of global structure, makes them particularly interesting and challenging in the digital humanities domain, both for ICT researchers who have the ambition of proposing tools to better organize and develop them, and for SSH (Social Sciences and Humanities) researchers who can benefit from explanatory models of historical evidence that may enrich narrative and even lead to new, plausible ones. This observation can be illustrated by the growing popularity of national and international initiatives, such as E-RIHS ${ }^{1}$ (European Research Infrastructure for Heritage Science) or the LSRI (Large Scale Research Initiative) Time Machine ${ }^{2}$, that gather hundreds of institutions in Europe.

Many of those contents can be associated, directly or indirectly, with a spatial information, from a simple street name to a position and orientation in 3D space. This information provides a common framework for their analysis and exploration, and may facilitate their exploration through time. Today, however, solutions allowing the joint exploitation of these spatialized data remain rather basic, most of the time it is a question of visualizing them on a 2D map with a GIS, layer by layer. In this context, the main contribution of the work presented here relates to the proposal of a web platform able to centralize different modalities of data and integrating several spatio-temporal visualization tools in a 3D environment, with the objective of enlightening the reading of sociologists and historians, and even to bring

\footnotetext{
1 http://www.erihs.fr

2 https://www.timemachine.eu/
}

new points of view, in the joint interpretation of the data manipulated. The work presented is multidisciplinary, bringing together ICT researchers specialized in geographic information, historians and sociologists in urban visual sociology. It is evaluated and illustrated here with the understanding of the construction of the Parisian metropolis, in particular through a very changing French city, Nanterre and its former shantytowns.

The article is organized as follows: section 2 introduces the data that are considered here, before revisiting the state of the art in visualization of georeferenced information in section 3 . Then we present our web-based proposal for spatio-temporal visualization of heterogeneous multi-date data in 3D within section 4 , before explaining its exploitation in support of two use cases focused on understanding the formation of the Parisian metropolis in section 5 and concluding in the last section.

\section{DATA}

With the development of powerful scanning tools and the availability of storage and sharing infrastructures, more and more digitized or digital data are made available. In this study, we focus on statistical data related to census and on visual data provided by aerial imagery; they are both described in section 2.1 . To be registered in a common 3D reference space, these data have to be geolocalized. In section 2.2, we revisit the different kinds of information available for their 3D localization.

\subsection{Statistical and visual data}

2.1.1 Census as statistical data. General population censuses, which have been held at more or less regular intervals in France since the beginning of the 19th century, are an invaluable source of knowledge for demographers, historians and sociologists. The results are presented in the form of aggregated tables at several administrative scales (country, regions, departments, etc.). "It was in 1946 that the "îlot" (administrative cluster) was first used systematically as a basic census unit for the City of Paris. In 1954, its use was extended to most of the suburban communes of the Seine. (...) The "îlot" is defined as the smallest part of the communal territory, 
entirely delimited by public or private communal roads" 3 ; Figure 1 shows them for Nanterre.

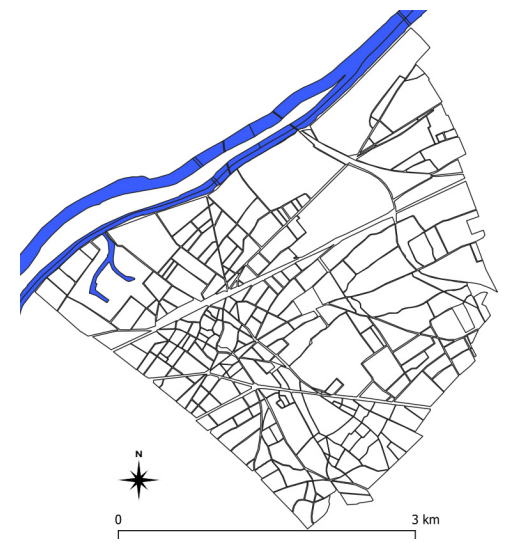

Figure 1. Census as statistical data: Focus on Nanterre and its "îlots" in 1954.

In 1954, we have four imposing volumes (roughly 1700 pages in total) giving us a mine of information for 71 communes of the former department of the Seine, to which it is necessary to add another volume for Paris.

For a reason unknown to us, 8 of the 81 communes of the department are missing. For each municipality, four tables deal successively with the population, the working population, housing and buildings. In total, a multitude of variables are shown, which allows a wide variety of aspects to be addressed in a finely tuned spatial perspective. The first table concerns the population. In addition to the distribution by gender and age, the census indicates the number of "Muslims of Algeria" for each block; Figure 9 illustrates them for Nanterre city. The second table,that of the working population, shows the distribution of the working population according to the new socioprofessional categories devised by INSEE in 1954. Individuals are classified according to their professional situation, taking into account several criteria: the profession itself, economic activity, qualification, hierarchical position and status. The third table gives information on dwelling units and their occupancy and equipment. It shows how many dwellings had running water or central heating in each block. The number of dwellings in acceptable or critical overcrowding situations is counted. The last table concerns the built environment. This makes it possible to identify the nature of the buildings (dwelling houses, hotels, etc.), the date of construction or the number of floors.

2.1.2 Oblique imagery as visual data. The data considered are aerial imagery about the French territory at several time periods, made available by the French mapping agency (IGN) and the Departmental Archives of Hauts de Seine area (AD92). Since the 1920s, IGN has performed regular surveys covering the territory with vertical imagery, as well as other acquisitions, through oblique imagery, targeted on particular areas such as those that have undergone significant changes over time (destructions, reconstructions, etc.). Vertical views make it easy to situate oneself, their observation is almost similar to the reproduction found in maps. Contrary to oblique images, they offer an exhaustive overview of the territory. By contrast, oblique views offer a better perception and understanding of the topography, objects, architectures and local areas, since its angle is closer to the representation made by our eyes, with a shot generally taken at a lower altitude (Humbert et al., 2013). In this

\footnotetext{
3 Prefaces to the 1954 Census data tables by "îlots". The preface is identical for each commune.
}

article, we choose to focus on this latter group, whose characteristics may be particularly relevant for historical and sociological analysis. The collection considered is about 30000 digitized photographs in total, about 700 for Nanterre area (illustrated in Figure 2) and about 200 for its shantytowns. Many other collections exist, usually hosted in GLAMs (e.g. Archives nationales for France).

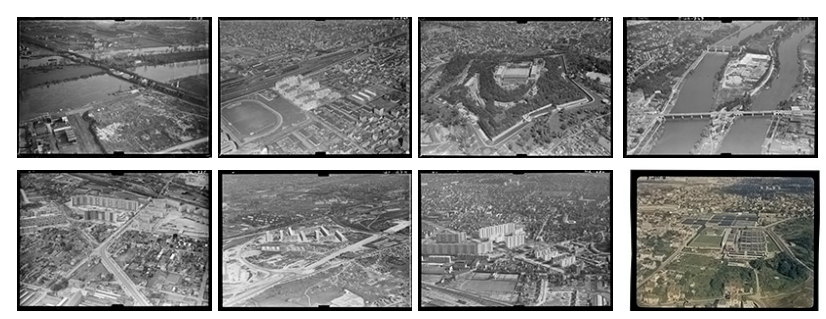

Figure 2. Some examples of IGN aerial oblique imagery: area of Nanterre, period 1947-1960 (CIGN)

\subsection{Different levels of localization information}

Classically, the census data were spatialized in a GIS. For each municipality, a plan of the "îlots" is provided in the volumes. They were digitized, then georeferenced (Lambert 93 projection) and finally vectorized using the QGIS software.

Positioning the image of the collections in a $3 \mathrm{D}$ environment supposes the knowledge of an information of localization. Here, such contents are associated with different levels of geolocalization, with which we have to deal with:

- Textual metadata providing an information of geolocalization through toponyms: department, city, locality, etc. It is often the case with collections from preservation institutions, such as the ones of AD92 used here. Geocoding algorithms, together with reference datasets, are usually exploited to determine a $2 \mathrm{D}$ position related to the centroid of the area described;

- Historical pose estimations (i.e. position and orientation): it is for example the case with contents from official surveys performed by mapping agencies, such as those of IGN seen in Figure 2;

- No geolocalization: in this case, first localization can be performed manually or semi-automatically, through GUI tools and user interactions, such as in some advanced approaches revisited in sections 3 and 4.2. They usually estimate this information by manually providing a set of point correspondences in the image and in the 3D scene, before regressing the estimation with a "perspective-npoint"-like algorithm. In computer vision, photogrammetry and robotics domains, there exist more advanced techniques for estimating the pose automatically; we revisit them briefly below.

Image precise geolocalization estimation. By precise geolocalization of an image, here we mean the ability of automatically determining the position and orientation (6 degrees of freedom - 6-Dof) of the acquisition camera in the 3D environment. This estimation is a core problem that can be tackled by a large panel of approaches (Lowry et al., 2016) (Garcia-Fidalgo, Ortiz, 2015) (Brejcha, Cadik, 2017) (Piasco et al., 2018). If the environment where to localize the image is small (a street, a district, etc.), approaches based on the regression of the pose, today with deep networks, is popular, such as PoseNet (Kendall et al., 2015). when the area to cover is larger, image localization can be solved using content-based image retrieval (Arandjelović et al., 2017). From a reference environment described 
with a set of geolocalized images, a coarse pose can be obtained by retrieving the reference image the closest to the query, such as in (Song et al., 2016). The most successful approaches exploit all the available modalities, and match $2 \mathrm{D}$ image features to a 3D reference point cloud, before using a Perspective-nPoint $(\mathrm{PnP})$ algorithm to estimate the 6-DoF pose of the image (Sattler et al., 2018) (Taira et al., 2018).

\section{RELATED WORK}

This section gives an overview of the literature related to the visualization of heterogeneous data in a common georeferenced environment. Several web-based applications tackle the subject of geolocalizing images, even multi-date images, and visualizing them, interacting with them in a dedicated interface. Historically, many of them rely on a 2D environment, thus we revisit them first in section 3.1 before detailing those dedicated to 3D scenes in section 3.2.

\subsection{D-based platforms}

A first category of applications focuses on vertical aerial images such as orthomosaïcs. The project Planet Explorer ${ }^{4}$ allows users to visualize satellite images worldwide with different tools to compare them, such as timelapses or sliding comparisons between images. Similarly, several national mapping agencies provide web portals dedicated to the valorization of their historical data (mainly maps and aerial imagery), where usually the user can visualize, compare and even download them: e.g. the web portal "Remonter le temps" of the IGN that manages data from the $18^{\text {th }}$ century up to now; or the portal "Voyage dans le temps" of the Swiss mapping agency ${ }^{6}$. However, for both platforms, one can not load personal images, visualization is strictly in $2 \mathrm{D}$ and spatialization/registration of the data has been operated manually.

Another category concentrates on localizing various kinds of historical information such as images, maps, statistics or articles. The proposals are numerous, generally concentrated on a particular objective or application for a given community. One can mention Navigae ${ }^{7}$ that allows users to visualize images and maps next to a map where they are coarsely localized. Then comes the project of the research group Inventer le Grand Paris ${ }^{8}$ that simply localizes research projects about world metropolis at different periods with illustrative images. The platform becomes a spatio-temporal filter of those projects.

Lastly, other solutions focus on images and their visualization in a mixed 2D-3D fashion. First we mention the well-known Mapillary ${ }^{9}$ project. It localizes worldwide street-level images and panoramas with a smooth visualization process between close-by images. Differently, Navilium ${ }^{10}$ is a collaborative platform with localized images from the $19^{\text {th }}$ century. If available, it is possible to visually compare the historical image localized in 2D with current StreetView images at the same position at the same position in a side-by-side fashion. We also mention the project HistoryPin ${ }^{11}$ that gathers historical iconographic collections by theme or location and "pins" the images on a 2D map. An interesting feature is the possibility of viewing the image in Google StreetView, provided that the image

\footnotetext{
4 https://www.planet.com/explorer/

5 https://remonterletemps.ign.fr/

6 https://www.geo.admin.ch

https://www.navigae.fr/

http://www.inventerlegrandparis.fr/

9 https://www.mapillary.com/

10 https://www. navilium.com/

11 https: //www.historypin.org/
}

was taken at street level. The estimation of the pose of the image in StreetView is rudimentary, as it is simply a manual superposition of the image on a given StreetView camera position. Hence, we obtain a pseudo- $6 \mathrm{DoF}$ pose of the image as it depends on one precise camera position in StreetView. Furthermore, the subsequent visualization of the image requires of the user that he does not move the camera around, otherwise losing the initial (correct) position of the image that follows the camera as it moves. Finally, the project ArchiMediaL ${ }^{12}$ works toward automatic linking of image content for comparative investigation of contemporary and historic built forms. To this end, an annotation platform ${ }^{13}$ of historical images is proposed, partly based on Mapillary's visualizer, in order to enrich the data content with semantic information. Hence images are registered as street-view or interior for instance. Furthermore, if changes have occurred, they are semantically registered ("building destruction", "new building built", etc.). Such images can be used in conjunction with other historical data to help researchers in various fields. However, the platform can not visualize other kinds of past data, other means of visualization for the other data types are necessary to complete this platform.

\subsection{D-based platforms}

The number of online applications involving a 3D environment is smaller. The exploitation of the third spatial dimension can be naturally related to the nature of the data being manipulated, e.g. three-dimensional architectural objects. This is the case with the Aioli ${ }^{14}$ collaborative platform that aims to document and share our architectural cultural heritage. Using several visualization processes, both $2 \mathrm{D}$ and $3 \mathrm{D}$, it allows users to annotate on images or 3D models of heritage objects. The added data is then propagated to all users and can even broaden the annotations on similar images automatically using photogrammetric techniques. Multi-temporal navigation is also available so as to visualize the heritage object at different periods, in order to check its conservation process or on the contrary to identify possible degradation.

UrbanHistory $4 \mathrm{D}^{15}$ is another $3 \mathrm{D}$ visualizer, dedicated to the restitution or urban heritage environments, currently implemented on the city of Dresden, Germany (Maiwald et al., 2019). Historical iconography (paintings, sketches, photographs), from 1675 and from various collections, is precisely positioned and oriented in a detailed 3D model of a district of the city, using an ad hoc Structure-from-Motion procedure. When visualizing images, one can also set the camera as the one taking the picture, to see the image in the context of the $3 \mathrm{D}$ building model. Several interesting visualization paradigms are available such as a heat map of the spatial distribution of the iconography, a radar chart of the orientations, etc. However, the user cannot upload any of his own images to geolocalize them and the current version of the system appears to be calibrated for one given limited area of study.

Smapshot (Blanc et al., 2018) is a Swiss web-based participatory virtual globe where users participate in georeferencing historical images of the landscape provided either by the Swiss ETH library or the Swiss Federal Office of Topography, by manually clicking a minimum of six well identifiable correspondence points between the image and the $3 \mathrm{D}$ space. The users must have a basic understanding of geographic notions as they should first specify the geographical position of the photography on the map, then define the shooting direction, and

\footnotetext{
12 http://archimedial.net/

${ }^{13}$ http://archimedial.eu/beeldbank/login.php

${ }^{14}$ http://www.aioli.cloud/

15 http: //4dbrowser .urbanhistory4d.org/
} 
lastly identify the correspondences. Smapshot is concentrated on the swiss territory and it relies on the Image Archive team to validate the image after the user have successfully localized the image.

Finally, (Chagnaud et al., 2016) propose a solution for the visualization of documented 3D Cities relying on CityGML to reference documents on buildings.

\section{ONLINE VISUALIZATION PLATFORM}

In this section, we present the web-based application we propose for the co-visualization of spatialized multi-date data. In section 4.1 , we begin by revisiting iTowns, the open source web-based library used as 3D framework. Sections below introduce the new functionalities proposed to improve the experience in the managing of multi-date images: section 4.2 is dedicated to the presentation of an interactive tool for the precise geolocalization of images, while section 4.3 presents several solutions for spatio-temporal navigation and section 4.4 goes deeper with more advanced tools.

\subsection{An immersive web application for visualizing hetero- geneous data}

The system presented in this article is an interactive web application that allows users to browse through and navigate within different types of images (aerial oblique or vertical, terrestrial) potentially acquired at different time periods. They are projected into a common 3D reference model (representing the earth using up-to-date data).

The geospatial web application supporting the visualization and interaction tools is iTowns ${ }^{16}$, an open source library for the web (Devaux et al., 2012). It has been developed since 2008 and its core has proven its scalability by acting as 3D vizualization engine in the geoportal of the French mapping agency. With access to geographic web services, it can render the earth with heterogeneous data, such as aerial and terrestrial imagery (image and LiDAR), as well as vector data (terrain, building, etc.) with a high precision and at large scale. Another interesting feature is that it can render street-level imagery without any change in the reference. Hence, it is particularly appropriate as the 3D reference frame for our proposal.

In this reference frame, the background maps available are numerous, allowing to choose the best representation of the area of study to bring out the data that have to be visualized. Alongside images, vector data can also be projected on the 3D reference model. This other type of data brings new information on the area of study; it may simply be administrative boundaries but it can also be more complex statistics, as those presented in section 2. Figure 3 illustrates this powerful functionality, with the co-visualization of various types of data.

\subsection{Web-based interactive image geolocalization}

Because some of the contents managed here rely on historical photographs potentially not precisely localized in the 3D scene, one important functionality of the system is based on their geolocalization. We have adopted the semi-automatic approach previously proposed in (Harrach et al., 2019). Localization is achieved with 2D-3D registration by adopting an interactive approach where the user identifies at least 4 corresponding points on the image to localize and the 3D reference model. This 3D model is at least a Digital Elevation Model in order to create the

\footnotetext{
${ }^{16}$ https ://github.com/itowns/itowns
}

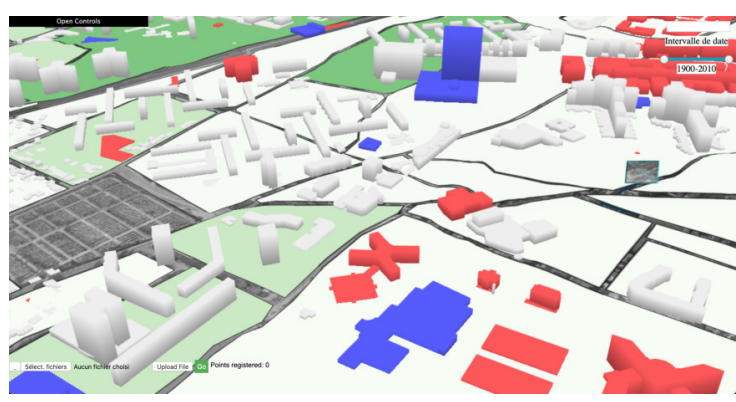

Figure 3. Co-visualization of 3D buildings (red: commercial, blue: public and remarkable, white: undifferentiated), statistical vector data, geolocalized oblique images and orthoimages.

whole virtual globe. However additional 3D information may be added, such as Digital Surface Model or 3D point clouds for instance. Here, we exploit a 3D representation of the buildings in order to improve the accuracy of our geolocalization. The interactive process of selection is illustrated in Figure 4.

The matches obtained are then used as input to a photogrammetric software that estimates the pose of the image. Mathematically speaking, the process consists in positioning a photograph using a cloud of 3D points or a photogrammetric model created via Structure-from-Motion. To this end, we use spatial resection within 3D "architectural" scenes (Busayarat, 2006). Space resection uses fixed object space coordinates and image coordinates of those objects to determine the camera's (or a photograph's) pose parameters: 3 position coordinates and 3 rotation angles (Moffitt, Mikhail, 1980). To solve this non linear problem, existing methods (Easa, 2010) consist in linearizing the collinearity condition and using the least squares method to iteratively determine the final solution. To initiate the process, approximate initial values are required, and can be estimated by the user as he/she positions him/herself roughly at the same position of the camera that took the photograph. More automated solutions focusing on the initial pose estimation are also used, as presented in section 2.2, using for instance the pose of the most similar image, or a combination of the poses of the most similar images (Song et al., 2016).

\subsection{An immersive navigation through space and time}

As the ambition is to co-visualize a great number of images (vertical, oblique and terrestrial), 3D models and $2 \mathrm{D}$ vector data, each having their own geolocalization and date, it is crucial to be able to navigate through space and time in an orderly, intuitive and ergonomic fashion.

4.3.1 Publishing massive georeferenced image datasets. To support massive image datasets, image poses and urls are streamed with space (bounding box) and time (interval) filters, using a standard webservice. Only images of interest are thereby downloaded, based on the user's location and time selection, ensuring smooth navigation and low bandwith. In practice, images are published using a Web Feature Service (WFS) of points, where the point geometry is the $3 \mathrm{D}$ camera location and the other camera parameters and image content url are provided as feature attributes. This way, image content is not duplicated but fetched from the conservation institution, preventing copy of its images from going around unchecked. The conservation institution only needs to suitably setup its cross origin (CORS) policy in order to authorize specific accesses.

4.3.2 Navigation through time. To navigate through time, a two-handle slider has been added to allow the user to select 


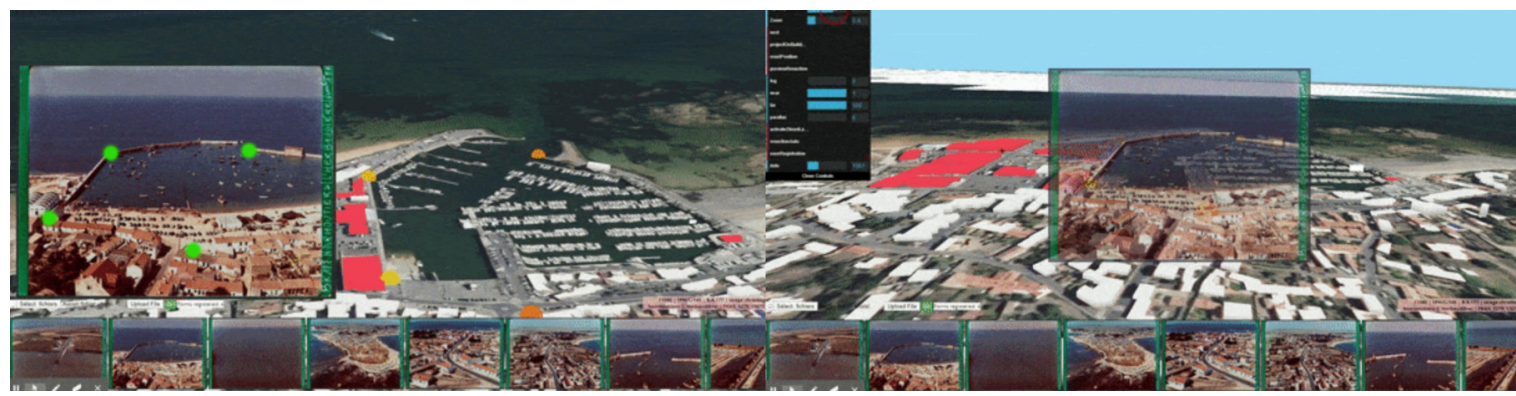

Figure 4. Semi-automatic localization of an image with iTowns: (left) Image registration with 4 pairs of user inputs (green points on the source image and orange points in the 3D reference. (right) Registered image in the 3D scene.

the time period considered in the data to visualize. All types of data are impacted by this choice as long as a date is provided for them. This allows to focus on a precise period and visualize the data available without the cluttering of other available data. This is a necessary tool required by historians and sociologists for historical studies as it allows to focus on certain dates. As use cases in Section 5 can attest to, historical and sociological studies may be restricted to tight time frames and sorting quickly through the data is paramount.

4.3.3 Navigation between points of view. To easily focus on the area of interest, the application integrates a geocoder in order to center the camera on a particular location provided through textual information: the user can propose a toponym (address, city name, etc.) where he/she wants to go and a smooth transition simulates the motion until the selected area, displaying the desired data simultaneously.

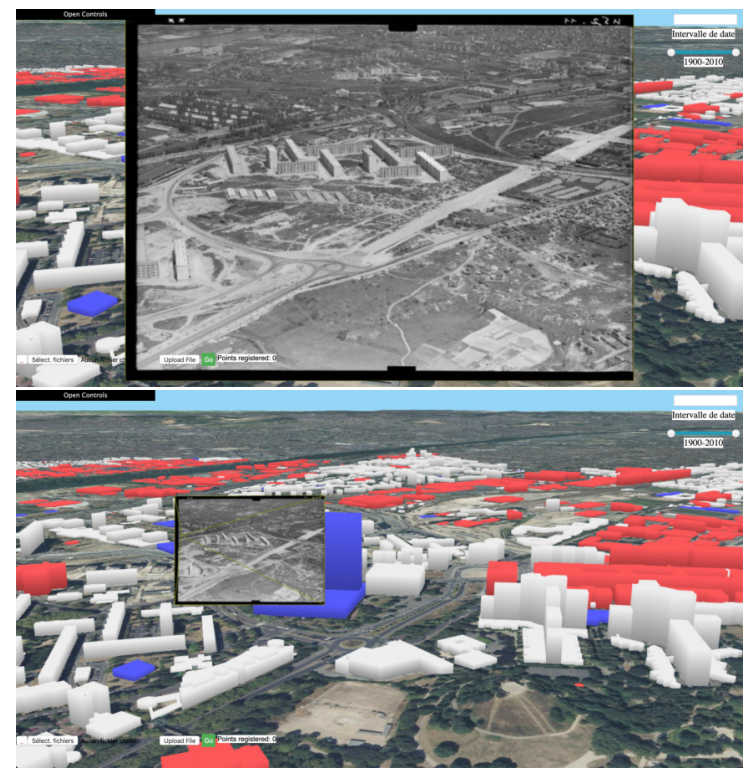

Figure 5. Navigation between points of view: oblique image seen from a camera viewpoint (top) or a free viewpoint (bottom).

Navigating through the data in the immersive environment is very intuitive as the user chooses between two different types of point of view, illustrated in Figure 5: either a free point of view, or the point of view of the camera that took the image of interest. The free point of view enables to roam free in the whole visualizer with the geolocalized images appearing oriented from the center of the camera that took them. One can also choose to set the point of view as the position of a camera that took a chosen picture.
4.3.4 Navigation via the image collection. To browse easily through the images of the collection, a carousel has been added at the bottom of the web browser in order to display a gallery of images: it displays images that are present on the area visualized ; see an example in Figure 6. While many images may be present in the same displayed area, the carousel can only show a limited subset, making available the rest by scrolling horizontally.

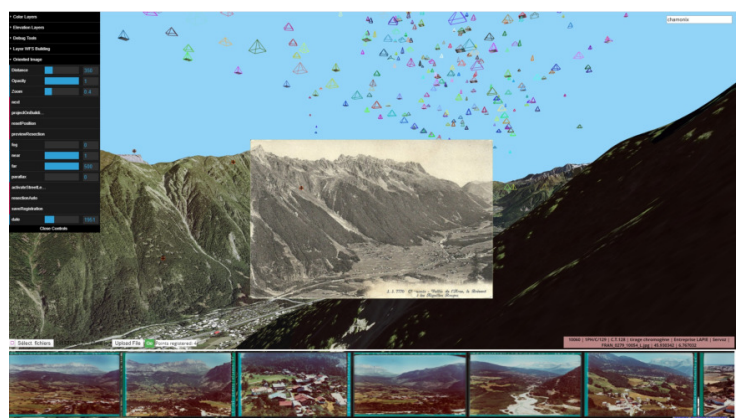

Figure 6. Illustration of the carousel providing the images available as clickable pyramids in the $3 \mathrm{D}$ visible environment (C)J. J. 7770 Chamonix - Vallée de l'Arve, le Brévent et les Aiguilles Rouges Jullien frères, Phot. Editeurs, Genève).

These images may be accurately geolocalized or just geocoded. By clicking on one image, if its precise pose is known, then the camera will center itself on the position of the image's camera in the scene. Otherwise, the image will be ready for registration in order to compute its precise pose and update the database. If the study is done on a specific area, as in the uses cases presented in section 5, it may be interesting to find images of the collection that show a specific area: a tool was then implemented in order to be able to find all precisely geolocalized images that can see a given point. Thus, once the point is chosen by manually clicking in the 3D scene, only images "seeing" this point are displayed in the 3D environment and the carousel will be updated to show only these images. Combined with the time frame tool, this tool allows to quickly sift through the image collection and find those of interest.

\subsection{Advanced visualization and interaction tools}

In this section, we go deeper by introducing some advanced paradigms of visualization and interaction.

4.4.1 Interactions between the content and the environment. To better experiment the relations between the contents and the 3D environment, it is possible to display them according to several modalities: 


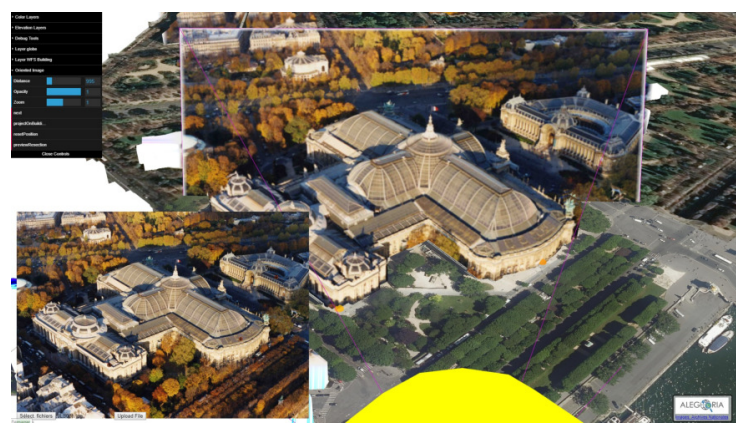

Figure 7. Projection of a geolocalized image in the 3D environment where 3D buildings are present and modeled.

- Playing with the image transparency in the environment, as illustrated in Figure 12;

- Zooming in and out of the scene, to see the image in its 3D context, possibly handling the geometric distortion present in the historical image (Paiz Reyes et al., 2020);

- Beyond simple blending, the image may be projected as a texture on the 3D scene (Brédif, 2013) to enable viewpoint displacements out of the image locations and selective texturing (buildings are textured in the example of Figure 7).

4.4.2 Global visualization paradigm. We can visualize the images as individual cameras, but the global setting of the viewer allows for a multi-scale visualization paradigm, in order to offer scalability during visualization and navigation. Visualizing the distribution of the collection at a glance, or by clusters, enables to easily concentrate on particular areas sufficiently furnished. We have implemented this functionality with heat maps; an example of the visualization process is presented in Figure 8, with transparency in order to visualize the map underneath for a better identification of the areas of interest.

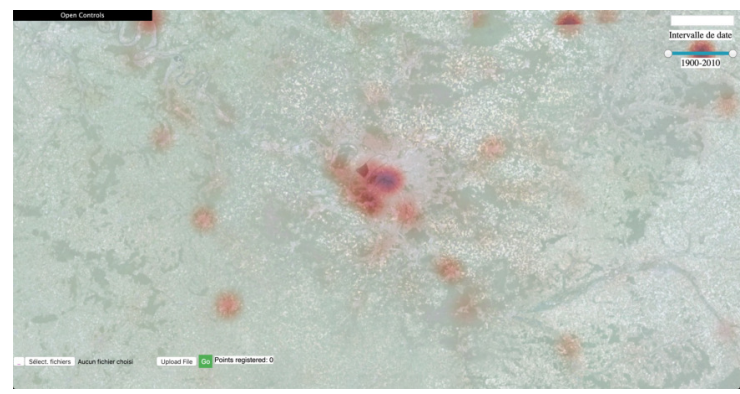

Figure 8. Multi-scale visualization of localized images with heat map, over Paris area.

\section{TWO USE CASES}

In this section, we present two distinct use cases of the exploitation of the web application presented in section 4 involving the data of section 2, as tool for analysis and interpretation for historians (section 5.1) and sociologists (section 5.2).

\subsection{History of metropolization phenomena in the Paris metropolitan area in the middle of the 20th century}

Exploiting the web-based co-visualisation tool, a preliminary study on the transformations of the city of Nanterre in the 1950s and 1960s was carried out. The aim was to better understand the formation of shantytowns during this period. By definition, their construction leaves few traces in the archives and it is very difficult to document the instant of their formation. But we have experimented that cross-referencing censuses and aerial views provides a better understanding of these phenomena.

Nanterre is a municipality on the outskirts of Paris. It underwent immense transformations throughout the early 20th century, following the urban expansion and the industrialization of the Parisian agglomeration. In 1954, Nanterre counted 53,011 inhabitants, which is almost four times higher than the population of the municipality in 1900 (14,140 inhabitants). The extension of the city was largely due to the construction of poor quality individual housing around the city centre and its railway station. Along the Seine river, the land is taken over by various industries. 1954's census testifies to these transformations with $62 \%$ of the population defined as working class.

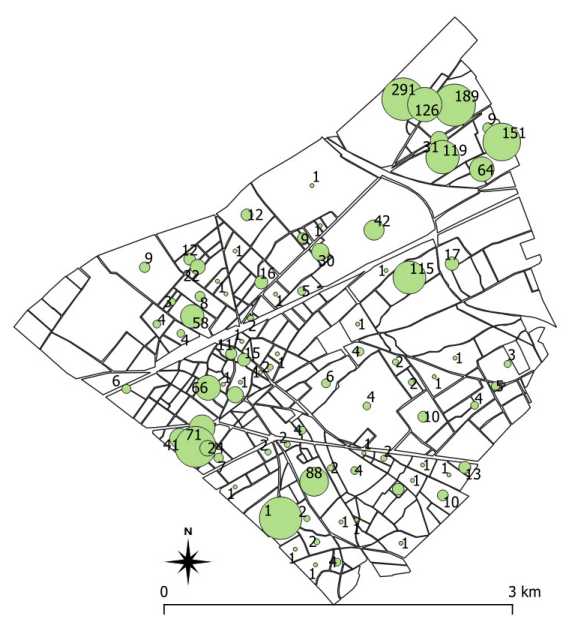

Figure 9. Distribution of the Algerian Muslims population in the "îlots" of Nanterre.

In 1954, Nanterre was on the eve of major changes as, in the 1950s and 1960s, Nanterre became a key location in the Parisian agglomeration with the installation of a large university, annex of the Sorbonne, and its designation as the capital of the new department of Hauts-de-Seine which was accompanied by the construction of a new prefecture. In addition, from 1951, under the impetus of the communist mayor, thousands of social housing units were built. All these transformations took place away from the old village in the north of the municipality and constituted a pole embodying the urban modernity of that time. However, at the same time, dozens of informal housing areas and shantytowns developed, sheltering mostly Algerians who had recently arrived in the metropolis. In the 1954 census, one can find a category developed by the colonial administration, called "Muslims of Algeria", as illustrated in Figure 9.

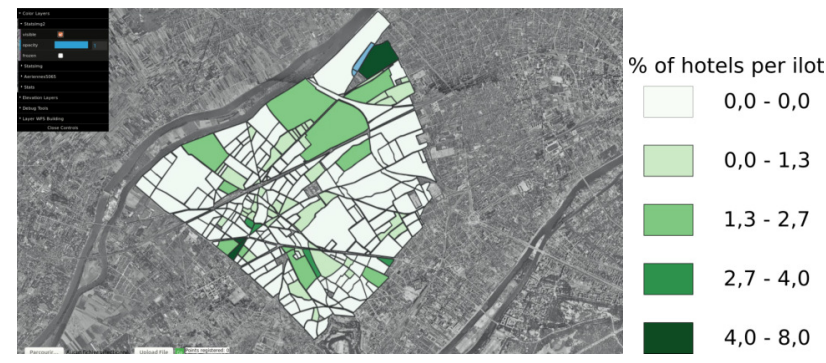

Figure 10. 3D navigation, with visualization of statistics (îlot scale) on hotels concentration in Nanterre area. 
It shows that the Algerians are concentrated on the northern part of the city, still little urbanized but already invested by many factories. Many of them stayed in small hotels which soon became overcrowded and therefore, from 1955 onwards, began to build shacks that would eventually become real shantytowns. Figure 10 shows the associated statistics within navigation in the $3 \mathrm{D}$ environment with iTowns.

In order to understand the constitution of these shantytowns, a census is of little use. It gives a good picture of the social and urban state in 1954 but to perceive a diachronic dimension, one has to turn to aerial photographs that allow us to see the evolution of the landscape. By navigating into this data through the immersive environment presented in section 4 , we can see that the first shantytown, called "La Folie", did not form on virgin land as one might have thought, but in parallel with the construction of the first large social real estate housing. Figure 11 illustrates the navigation performed.

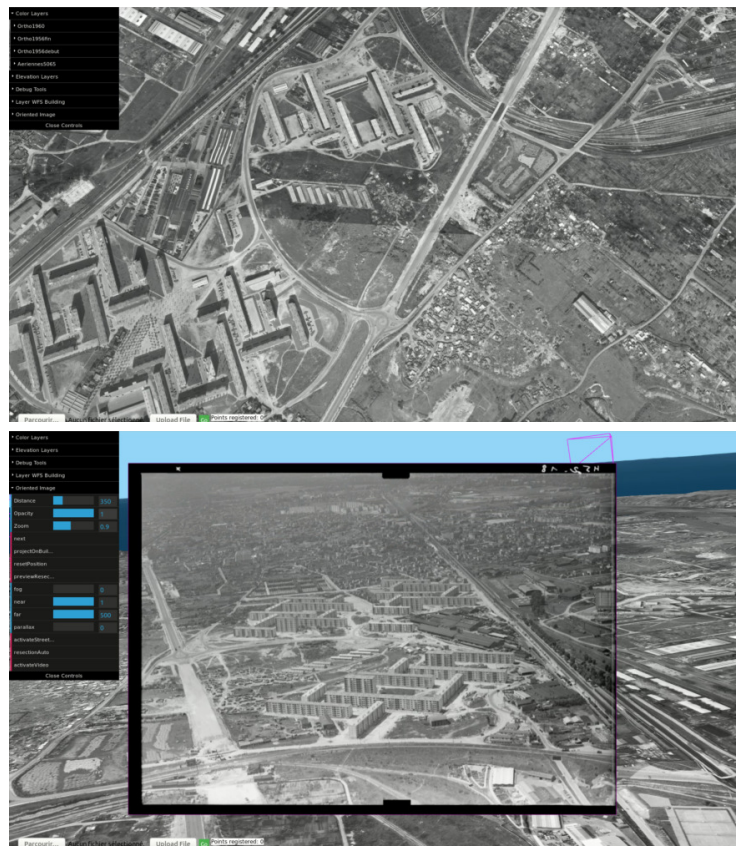

Figure 11. Navigation in the 3D environment: (top) Vertical view, with historical ortho-imagery, of the shantytowns next to a brand new housing complex; (bottom) another point of view, through historical geolocalized oblique views.

From these aerial photographs, it is possible to hypothesize that the first shantytowns were used as housing for the construction workers hired for the large building sites of Nanterre. These symbols of the urban modernity of the "trente glorieuses" hence produced urban forms that are actually considered to be the antithesis of the modern city.

\subsection{Study of modes of representation of social changes in the inhabited space in urban visual sociology}

Having access to a large number of photographs may improve research work in visual sociology concerning urban environments in Europe and more specifically the modes of representation of social changes in inhabited space, such as in the city of Nanterre where many changes occurred during the 20th century. While Nanterre, like its shantytowns, is historically rich, the association of photographs and testimonies of Nanterre's inhabitants opens a new perspective: the use of photoelicitation from aerial images. Introduced by the American anthropologist-photographer John Collier in 1967, this method has since been taken up in sociology in various works such as (Duteil-Ogata, 2007). It consists in using photographic image as a medium for discourse during interviews in the research field, as here with inhabitants of Nanterre, or former shantytown dwellers. It opens up a perspective of looking at the representations of the interviewees and thus understanding, in the case of aerial photographs, the modes of perception of the spatial dimension, and by deepening the interview, the history of the city and its changes over time experienced by the individuals.

In this work, our objective is to evaluate how the proposed web application built using iTowns can contribute to the sociological method of photo-elicitation interviewing for the geographical study of the transformation of the territory of Nanterre, with a focus on its shantytowns. We have exploited the collection of oblique photographs described in section 2.1.2. As preamble, a total of 174 non-localized oblique photographs of shantytowns and neighborhoods, from the AD92 collection, were geolocalized with success thanks to the semi-automatic approach proposed in section 4.2. To evaluate the web application in the context of photo-elicitation, three complementary profiles of people were identified to answer the interviews:

- An inhabitant of Nanterre, specialist in its history and geography for decades;

- Two inhabitants of the city, former inhabitants of one of the shantytowns;

- The director of the Nanterre tourism office, who does not live in the city and is just discovering its history.

To each profile, a protocol of interview was established: the sociologist has associated a series of photographs, as do dedicated interviews. Commonly, the images are selected according to a time period with the time selector (section 4.3.2) and displayed in the 3D environment together with additional data such as simple representations of buildings (similarly to those of Figure 5). Interactions with the system mainly consisted in moving from one viewpoint to another (including vertical viewpoints to compare their readability facing oblique ones), as well as exploiting the transparency tool (section 4.4.1) to exhibit the changes through time, as illustrated in Figure 12.

The interviewees, particularly the experts, were sometimes disoriented by certain limitations of the current version of the application, mainly due to the inaccuracy of some data representing the 3D environment: lack of texture on the buildings, vertical reference views dating back 2-3 years, imprecision of certain reliefs or skyline, etc. However, several aspects have raised interest and fostered discussions on the history and sociological aspects of the city, such as the possibility of observing the city from a bird's eye view, thus offering understandable details of areas while leaving visible visual cues necessary for a visitor's global orientation; or being able to compare areas undergoing major changes by interacting on the transparency of the views or the display of multi-date images. Whether for experts or non-specialists, the interest of the proposed tools for transmission purposes was considered undeniable, especially for younger generations or tourists

\section{CONCLUSIONS AND FUTURE WORK}

This article has presented an online visualization platform dedicated to visualizing heterogeneous data of the past. Several tools and visualization options were developed in order to improve the immersive experience and its use by researchers, with 

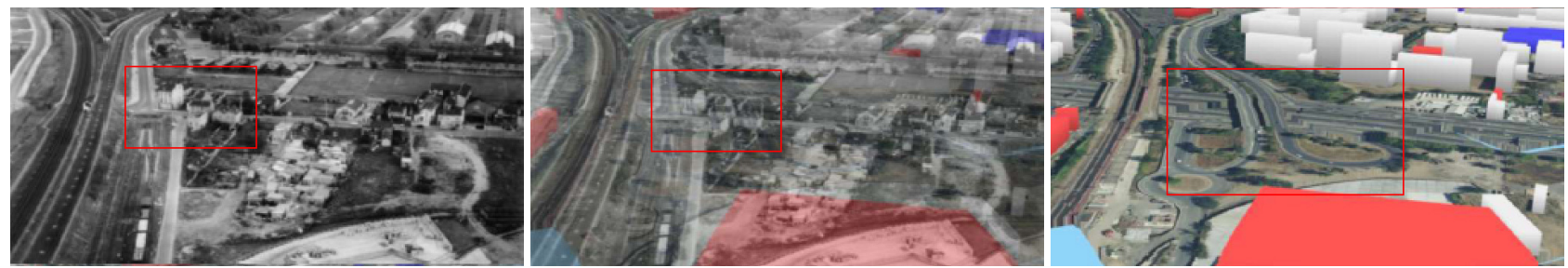

Figure 12. Interaction between a photograph (left) and the 3D scene (right) through transparency (middle). Infrastructure evolution and (dis)appearance of landmarks (hotel/restaurant vs highway interchange) are highlighted (C)AD92, period 1960-1961).

a focus on the digital humanities community. The two use cases considered have involved different protocols of navigation and interactions, with different objectives. Through these experiments, our group of multidisciplinary researchers have experimented with success the potential of the proposed web application, based on the sharing and handling of historical information with advanced ICT tools. The practicality of the global system highly relies on the quality of the data available for the $3 \mathrm{D}$ reference framework, and several other improvements and extensions are to be considered in the future, e.g.: integration of a full automatic image localization method; opening the platform to participatory functionalities to enrich the virtual environment with new contents and annotations. From the user perspective, larger studies will be considered, such as comparisons between municipalities and with international metropolises, with the aim of continuing improving the understanding of the formation of the Parisian metropolis in the middle of the 20th century.

\section{ACKNOWLEDGEMENTS}

The authors would like to thank Ordnance Survey GB (https://www.ordnancesurvey.co.uk) and 1Spatial (https://1spatial.com/) for sponsoring the publication of this paper. This work is supported by I-SITE FUTURE and ANR (French National Research Agency) respectively within the Archival City Tremplin project, and the ALEGORIA project under Grant ANR-17-CE38-0014-01. We also would like to thank Martyna Poreba and Nathalie Abadie for their help in making available the images of Nanterre and Benjamin Lecat for his work on the census data.

\section{REFERENCES}

Arandjelović, R., Gronat, P., Torii, A., Pajdla, T., Sivic, J., 2017. NetVLAD: CNN architecture for weakly supervised place recognition. IEEE Transactions on Pattern Analysis and Machine Intelligence (TPAMI), 5297-5307.

Blanc, N., Produit, T., Ingensand, J., 2018. A semi-automatic tool to georeference historical landscape images. PeerJ PrePrints, 6, e27204.

Brédif, M., 2013. Image-Based Rendering of LOD1 3D City Models for traffic-augmented Immersive Street-view Navigation. City Models, Roads and Traffic workshop (CMRT13), International Annals of the Photogrammetry, Remote Sensing and Spatial Information Sciences (ISPRS Annals), II-3/W3, Antalya, Turkey, 7-11.

Brejcha, J., Cadik, M., 2017. GeoPose3K: Mountain Landscape Dataset for Camera Pose Estimation in Outdoor Environments. Image and Vision Computing.

Busayarat, C., 2006. Conception d'un système de référencement spatial de photographie de relevé. MIAjournal, 1-6.

Chagnaud, C., Samuel, J. S., Servigne, S., Gesquière, G., 2016. Visualization of Documented 3D Cities. The Eurographics Workshop on Urban Data Modelling and Visualisation, Proceedings, Liège, Belgium, 87-93.
Devaux, A., Brédif, M., Paparoditis, N., 2012. A web-based 3D mapping application using WebGL allowing interaction with images, point clouds and models. 20th ACM SIGSPATIAL International Conference on Advances in Geographic Information Systems. ACM-GIS'12, isbn: 9781-4503-1691-0, ACM, ACM Press, 586-588.

Duteil-Ogata, F., 2007. La photo-interview : dialogues avec des Japonais. Ethnologie française, I, 69-79.

Easa, S. M., 2010. Space resection in photogrammetry using collinearity condition without linearisation. Survey Review, 42(315), 40-49.

Garcia-Fidalgo, E., Ortiz, A., 2015. Vision-based topological mapping and localization methods: A survey. Robotics and Autonomous Systems (RAS), 64, 1-20.

Harrach, M., Devaux, A., Brédif, M., 2019. interactive image geolocalization in an immersive web application. ISPRS - International Archives of the Photogrammetry, Remote Sensing and Spatial Information Sciences, XLII-2/W9, 377-380.

Humbert, A., Courtot, R., Renard, C., 2013. Landscapes from the sky, the interest of aerial photography. Journal of Mediterranean geography, 120, 111-126.

Kendall, A., Grimes, M., Cipolla, R., 2015. Posenet: A convolutional network for real-time 6-dof camera relocalization. ICCV, IEEE Computer Society, 2938-2946.

Lowry, S., Sünderhauf, N., Newman, P., Leonard, J. J., Cox, D., Corke, P., Milford, M. J., 2016. Visual Place Recognition: A Survey. IEEE Transactions on Robotics (TRO), 32(1), 1-19.

Maiwald, F., Bruschke, J., Lehmann, C., Niebling, F., 2019. A 4D information system for the exploration of multitemporal images and maps using photogrammetry, Web technologies and VR/AR. Virtual Archaeology Review, 10, 1 .

Moffitt, F. H., Mikhail, E. M., 1980. Photogrammetry. Harper \& Row.

Paiz Reyes, E., Brédif, M., Christophe, S., 2020. Geometric distortion of historical images for 3D visualization. ISPRS Congress, International Annals of the Photogrammetry, Remote Sensing and Spatial Information Sciences, Nice, France.

Piasco, N., Sidibé, D., Demonceaux, C., Gouet-Brunet, V., 2018. A survey on Visual-Based Localization: On the benefit of heterogeneous data. Pattern Recognition, 74, 90 - 109.

Sattler, T., Maddern, W., Torii, A., Sivic, J., Pajdla, T., Pollefeys, M., Okutomi, M., 2018. Benchmarking 6DOF Urban Visual Localization in Changing Conditions. IEEE Conference on Computer Vision and Pattern Recognition (CVPR).

Song, Y., Chen, X., Wang, X., Zhang, Y., Li, J., 2016. 6-DOF Image Localization From Massive Geo-Tagged Reference Images. IEEE Transactions on Multimedia (ToM), 18(8), 1542-1554.

Taira, H., Okutomi, M., Sattler, T., Cimpoi, M., Pollefeys, M., Sivic, J., Pajdla, T., Torii, A., 2018. InLoc: Indoor Visual Localization with Dense Matching and View Synthesis. IEEE Conference on Computer Vision and Pattern Recognition (CVPR). 